\title{
Longitudinal and transverse scaling functions within the coherent density fluctuation model
}

\author{
A. N. Antonov, ${ }^{1}$ M. V. Ivanov, ${ }^{1}$ M. B. Barbaro, ${ }^{2}$ J. A. Caballero, ${ }^{3}$ and E. Moya de Guerra ${ }^{4,5}$ \\ ${ }^{1}$ Institute for Nuclear Research and Nuclear Energy, Bulgarian Academy of Sciences, BG-1784 Sofia, Bulgaria \\ ${ }^{2}$ Dipartimento di Fisica Teorica, Università di Torino and INFN, Sezione di Torino, Torino, Italy \\ ${ }^{3}$ Departamento de Física Atómica, Molecular y Nuclear, Universidad de Sevilla, E-41080 Sevilla, Spain \\ ${ }^{4}$ Instituto de Estructura de la Materia, CSIC, Serrano 123, E-28006 Madrid, Spain \\ ${ }^{5}$ Departamento de Fisica Atomica, Molecular y Nuclear, Facultad de Ciencias Fisicas, Universidad Complutense de Madrid, \\ E-28040 Madrid, Spain
}

(Received 2 December 2008; published 7 April 2009)

\begin{abstract}
We extend our previous description of the superscaling phenomenon in inclusive electron scattering within the coherent density fluctuation model (CDFM). This model is a natural extension to finite nuclei of the relativistic Fermi gas (RFG) model within which the scaling variable $\psi^{\prime}$ was introduced. In this work we propose a new modified CDFM approach to calculate the total, longitudinal, and transverse scaling functions built up from the hadronic tensor and the longitudinal and transverse response functions in the RFG model. We test the superscaling behavior of the new CDFM scaling functions by calculating the cross sections of electron scattering (in quasielastic and $\Delta$ regions for nuclei with $12 \leqslant A \leqslant 208$ at different energies and angles) and comparing to available experimental data. The new modified CDFM approach is extended to calculate charge-changing neutrino and antineutrino scattering on ${ }^{12} \mathrm{C}$ at $1 \mathrm{GeV}$ incident energy.
\end{abstract}

DOI: 10.1103/PhysRevC.79.044602

PACS number(s): 24.10.-i, 21.60.-n, 25.30.Fj, 25.30.Pt

\section{INTRODUCTION}

The relativistic Fermi gas (RFG) model in Refs. [1,2] has been the basis for defining the scaling variable $\psi^{\prime}$ and for introducing the first theoretical considerations of the superscaling phenomenon. Superscaling has been observed in inclusive electron scattering from nuclei (see, e.g., Refs. [1-4]). The term "superscaling" includes the scaling of the first and the second kind (independence of the reduced cross section on the momentum transfer $q$ and the mass number $A$, respectively) that have been seen at excitation energies below the quasielastic (QE) peak. These studies can be considered a part of more general investigations that follow the ideas of West [5] on scaling phenomena. They include also the studies of the related $y$-scaling in high-energy electron-nuclei scattering (e.g., Refs. [5-13]). It has been shown in both $y$ - and $\psi^{\prime}$-scaling analyses that the scaling function is sensitive to the high-momentum components of the spectral function and, consequently, to the tail of the nucleon momentum distributions $n(k)$. Thus, knowledge of the scaling function can provide important information about the dynamical ground-state properties of the nuclei. In the $\psi^{\prime}<-1$ region, superscaling is due to the specific highmomentum tail of $n(k)$ caused by short-range and tensor correlations, which is similar for all nuclei and which is in turn related to specific properties of the nucleon-nucleon $(N N)$ forces near the repulsive core. Even more, it has been shown in Ref. [14] that the behavior of the scaling function $f\left(\psi^{\prime}\right)$ for values of $\psi^{\prime}<-1$ depends on the particular form of the power-law asymptotic of $n(k)$ at large $k$ related to a corresponding behavior of the in-medium $N N$ forces around the core. The latter dependence together with the existing link between the asymptotic behavior of $n(k)$ and the $N N$ forces makes it possible to conclude that the inclusive QE electron scattering at $\psi^{\prime} \lesssim-1$ provides important information on the $N N$ forces in the nuclear medium.
Confirming the superscaling behavior of the world data on inclusive electron scattering, the analyses in Refs. [3,4] have shown the necessity of considering this phenomenon on the basis of a more complex dynamical picture of realistic finite nuclear systems beyond the RFG. Indeed, the scaling function in the RFG model is $f\left(\psi^{\prime}\right)=0$ for $\psi^{\prime} \leqslant-1$, whereas the experimental scaling function extracted from $\left(e, e^{\prime}\right)$ data extends up to $\psi^{\prime} \approx-2$, where the effects beyond the mean-field approximation become important. One such approach is the coherent density fluctuation model (CDFM; e.g., Refs. $[15,16])$, which is a natural extension of the Fermi gas model based on the generator coordinate method [17] and includes long-range correlations (LRC) of collective type. The QE scaling function $f\left(\psi^{\prime}\right)$ is deduced in the CDFM on the basis of realistic density and momentum distributions in nuclei and it agrees with the empirical data for the scaling function for negative values of $\psi^{\prime} \leqslant-1$ [18-22]. This agreement is related to the realistic high-momentum tail of the nucleon momentum distribution in the CDFM, which is similar for a wide range of nuclei, in contrast with the sharp behavior of $n(k)$ as a function of $k$ in the RFG model (see, e.g., Fig. 3 of Ref. [18], Fig. 2 of Ref. [19], and the analysis in Ref. [14]). A number of studies of superscaling have been published in the past decade or so (e.g., Refs. [23-35]). A "universal" phenomenological QE scaling function $f^{\mathrm{QE}}\left(\psi^{\prime}\right)$ has been obtained $[3,4,23,24]$ on the basis of the available separation of inclusive electron scattering data into their longitudinal and transverse contributions for nuclei with $A>4$. An unexpected feature of this scaling function extracted from the superscaling analyses (SuSA) is its asymmetric shape with respect to the peak position $\psi^{\prime}=0$ with a long tail extended toward positive $\psi^{\prime}$ values. This is in contrast to the RFG scaling function, which is symmetric. Detailed studies of this asymmetry [36-38] within the relativistic mean-field (RMF) approach have shown the important role played by a proper description of final-state 
interactions (FSI) in reproducing the asymmetric shape of $f\left(\psi^{\prime}\right)$. The existence of the asymmetric tail of the scaling function has also been shown recently in Ref. [35] in a model accounting for pairing BCS-type correlations.

The approach of SuSA to QE electron scattering has been extended in Ref. [24] to the $\Delta$-resonance excitation region.

The features of superscaling in inclusive electron-nucleus scattering have made it possible to initiate analyses of neutrino and antineutrino scattering off nuclei on the same basis (e.g., Refs. [24,36,39,40]). Neutrino-nucleus or antineutrinonucleus charge-changing (CC) [36] or neutral-current (NC) [32] scattering cross sections for intermediate to high energies can be calculated by multiplying the elementary singlenucleon $\mathrm{CC}$ or NC neutrino (or antineutrino) cross sections by the corresponding scaling functions. The assumptions leading to such a procedure have been tested within the RMF plus FSI model [41]. Here we mention a number of other theoretical studies of CC (see, e.g., Refs. [42-51]) and NC (e.g., Refs. [42,43,50,52-56]) neutrino- and antineutrinonucleus scattering in recent years.

The CDFM scaling function has been used to predict cross sections for several processes: inclusive electron scattering in the QE and $\Delta$ regions $[21,22]$ and neutrino (antineutrino) scattering both for $\mathrm{CC}$ [22] and for NC [56] processes ( $u$-channel inclusive processes). The CDFM analyses became useful to obtain information about the role of the nucleon momentum and density distributions for the explanation of superscaling in lepton-nucleus scattering [19,21]. It may also prove to be useful to explore the extension of the $u$ and $t$-channel scaling criteria beyond independent particle models.

It is important to point out that the physics contained in the "experimental" scaling function comes not only from the initial but also from the final states involved in the scattering process. Hence, caution should be placed on the general connection between the scaling function and the spectral function (or momentum distribution). Nevertheless, following the general consideration introduced in Ref. [14] on the relationship between $f\left(\psi^{\prime}\right)$ and the nucleon momentum distribution $n(k)$, it was found within the CDFM [19] that the slope of the QE scaling function $f\left(\psi^{\prime}\right)$ at negative values of $\psi^{\prime}$ crucially depends on the high-momentum tail of the momentum distribution $n(k)$ at larger values of $k(k>$ $\left.1.5 \mathrm{fm}^{-1}\right)$. Moreover, the sensitivity of the scaling function in the CDFM to the particular behavior of $n(k)$ in different regions of $k$ has been studied in Ref. [19], showing that the available empirical data on $f\left(\psi^{\prime}\right)$ are informative for $n(k)$ for momentum $k$ up to $k \leqslant 2-2.5 \mathrm{fm}^{-1}$.

In our previous works [18-21] we obtained the CDFM scaling function $f\left(\psi^{\prime}\right)$ starting from the RFG model scaling function $f_{\mathrm{RFG}}\left(\psi^{\prime}\right)$ and convoluting it with the weight function $|F(x)|^{2}$, which is related equivalently to either the density $\rho(r)$ or the momentum distribution $n(k)$ in nuclei. Thus, the CDFM scaling function is an infinite superposition of weighted RFG scaling functions. This approach improves upon RFG and enables one to describe the scaling function for realistic finite nuclear systems. In the approach in Refs. [18-21] the longitudinal and transverse scaling functions are equal
[ $f_{L}\left(\psi^{\prime}\right)=f_{T}\left(\psi^{\prime}\right)$, the so-called scaling of zero kind]; this is also a property of the RFG scaling functions. The aim of this work is to develop a new CDFM approach in which we start directly from the hadronic RFG [1] tensor $W^{\mu v}$ and the corresponding response functions $R_{L, T}$, and convolute them with the CDFM function $|F(x)|^{2}$. We call this new approach $\mathrm{CDFM}_{\mathrm{II}}$ to distinguish it from our former version to which we refer as $\mathrm{CDFM}_{\mathrm{I}}$. This method provides a more general way to apply CDFM ideas and to go beyond the RFG in the construction of scaling functions. Particularly, it allows us to study the possible violation of the zero-kind scaling $\left[f_{L}\left(\psi^{\prime}\right) \neq f_{T}\left(\psi^{\prime}\right)\right]$ and to compare the behavior of $f_{L}$ and $f_{T}$ to that from other approaches [e.g., the relativistic plane-wave impulse approximation (RPWIA)]. It can be seen in our work that the $\mathrm{CDFM}_{\mathrm{II}}$ scaling function calculated for different values of the transferred momentum $q$ shows both a saturation of its asymptotic behavior and also the region of appearance of the scaling of the first kind (at values of the transverse momentum of the order or higher than $0.5 \mathrm{GeV} / c$ ). The main difference between $\mathrm{CDFM}_{\mathrm{I}}$ and $\mathrm{CDFM}_{\mathrm{II}}$ is that in $\mathrm{CDFM}_{\mathrm{I}}$ the LRC are taken a posteriori in the scaling function, once the RFG scaling function has been derived from the total inclusive cross section, whereas in the $\mathrm{CDFM}_{\mathrm{II}}$ the correlations are included through the weighting function $|F(x)|^{2}$ in the hadronic tensor (i.e., they are included at an earlier stage in the derivation of the cross section). This allows us to study the emergence of scaling within the model, as well as possible differences between longitudinal and transverse scaling functions.

The second aim of the present work is to apply the obtained $\mathrm{CDFM}_{\mathrm{II}}$ scaling functions $\left(f, f_{L}\right.$, and $f_{T}$ ) to calculate cross sections of inclusive electron scattering off various nuclei, as well as cross sections of $\mathrm{CC}$ neutrino (antineutrino) scattering on ${ }^{12} \mathrm{C}$ at intermediate energies.

The theoretical scheme used in the present work is given in Sec. II. It includes the basic relationships of the RFG model for the hadronic tensor, the response functions, and the procedure to obtain the $\mathrm{CDFM}_{\mathrm{II}}$ scaling functions. The results for $f\left(\psi^{\prime}\right), f_{L}\left(\psi^{\prime}\right)$, and $f_{T}\left(\psi^{\prime}\right)$, as well as those from calculations of inclusive electron scattering cross sections in both $\left(\mathrm{CDFM}_{\mathrm{I}}\right.$ and $\left.\mathrm{CDFM}_{\mathrm{II}}\right)$ approaches and of cross sections of $\mathrm{CC}$ neutrino reactions on ${ }^{12} \mathrm{C}$, are presented and discussed in Sec. III. The conclusions are summarized in Sec. IV.

\section{THEORETICAL SCHEME}

We begin this section with a brief discussion of the basic formalism for inclusive electron scattering from nuclei [1] in which an electron with four-momentum $K^{\mu}=(\epsilon, \mathbf{k})$ is scattered through an angle $\theta=\measuredangle\left(\mathbf{k}, \mathbf{k}^{\prime}\right)$ to four-momentum $K^{\prime \mu}=\left(\epsilon^{\prime}, \mathbf{k}^{\prime}\right)$. The four-momentum transferred in the process is then $Q^{\mu}=\left(K-K^{\prime}\right)^{\mu}=(\omega, \mathbf{q})$, where $\omega=\epsilon-\epsilon^{\prime}, q=$ $|\mathbf{q}|=\mathbf{k}-\mathbf{k}^{\prime}$, and $Q^{2}=\omega^{2}-q^{2} \leqslant 0$. In the relativistic limit (ERL) $|\mathbf{k}| \cong \epsilon \gg m_{e}$ and $\left|\mathbf{k}^{\prime}\right| \cong \epsilon^{\prime} \gg m_{e}$, where $m_{e}$ is the electron mass. In the one-photon-exchange approximation, the double-differential cross section in the laboratory system can 
be written in the form

$$
\begin{aligned}
\frac{d^{2} \sigma}{d \Omega d \epsilon^{\prime}}= & \sigma_{M}\left[\left(\frac{Q^{2}}{q^{2}}\right)^{2} R_{L}(q, \omega)\right. \\
& \left.+\left(\frac{1}{2}\left|\frac{Q^{2}}{q^{2}}\right|+\tan ^{2} \frac{\theta}{2}\right) R_{T}(q, \omega)\right],
\end{aligned}
$$

where $L(T)$ refer to responses with longitudinal (transverse) projections (i.e., with respect to the momentum transfer direction) of the nuclear currents, and where the Mott cross section is given by

$$
\sigma_{M}=\left[\frac{\alpha \cos (\theta / 2)}{2 \epsilon \sin ^{2}(\theta / 2)}\right]^{2}
$$

with $\alpha$ the fine-structure constant.

This cross section is obtained by contracting leptonic and hadronic current-current interaction electromagnetic tensors and hence it is proportional to $\eta_{\mu \nu} W^{\mu \nu}$. The leptonic tensor $\eta_{\mu \nu}$ may be calculated in the standard way involving traces of Dirac $\gamma$ matrices and under ERL conditions becomes

$$
\eta_{\mu \nu}=K_{\mu} K_{v}^{\prime}+K_{\mu}^{\prime} K_{v}-g_{\mu \nu} K \cdot K^{\prime}
$$

Contracting this with a general hadronic tensor $W^{\mu \nu}$ and rewriting the cross section in Eq. (1), we have the following for the two response functions (using summation convention on repeated indices):

$$
\begin{aligned}
& R_{L}(q, \omega)=W^{00} \\
& R_{T}(q, \omega)=-\left(g_{i j}+\frac{q_{i} q_{j}}{q^{2}}\right) W^{i j} .
\end{aligned}
$$

In the RFG model the hadronic tensor $W^{\mu \nu}$ can be expressed by

$$
\begin{aligned}
W^{\mu \nu}= & \frac{3 \mathcal{N} m_{N}^{2}}{4 \pi p_{F}^{3}} \int \frac{d^{3} p}{E(\mathbf{p}) E(\mathbf{p}+\mathbf{q})} \theta\left(p_{F}-|\mathbf{p}|\right) \\
& \times \theta\left(|\mathbf{p}+\mathbf{q}|-p_{F}\right) \delta[\omega-[E(\mathbf{p}+\mathbf{q})-E(\mathbf{p})]] \\
& \times f^{\mu \nu}(P+Q, P), \mathcal{N}=N, Z,
\end{aligned}
$$

where the scattering is assumed to involve a struck nucleon of mass $m_{N}$ and four-momentum $P=[E(\mathbf{p}), \mathbf{p}]$ with corresponding (on-shell) energy $E(\mathbf{p})=\left(\mathbf{p}^{2}+m_{N}^{2}\right)^{1 / 2}$ lying below the Fermi momentum $p_{F}$ and having supplied energy and momentum $\omega$ and $q$, respectively, to the nucleon, resulting in a four-momentum $(P+Q)^{\mu}$ lying above the Fermi surface. $f^{\mu \nu}(P+Q, P)$ is the single-nucleon response tensor evaluated in the system where the struck nucleon has four-momentum $P$ :

$$
\begin{aligned}
f^{\mu \nu}(P+Q, P)= & -W_{1}(\tau)\left(g^{\mu \nu}-\frac{Q^{\mu} Q^{v}}{Q^{2}}\right) \\
& +W_{2}(\tau) \frac{1}{m_{N}^{2}}\left(P^{\mu}-\frac{P \cdot Q}{Q^{2}} Q^{\mu}\right) \\
& \times\left(P^{\nu}-\frac{P \cdot Q}{Q^{2}} Q^{\nu}\right) .
\end{aligned}
$$

Then the response functions in the RFG model can be written as

$$
\begin{aligned}
R_{L, T}^{(\mathrm{RFG})}= & \frac{3 \mathcal{N}}{4 m_{N} \kappa \eta_{F}^{3}}\left(\varepsilon_{F}-\Gamma\right) \Theta\left(\varepsilon_{F}-\Gamma\right) \\
& \times\left\{\begin{array}{l}
\frac{\kappa^{2}}{\tau}\left[(1+\tau) W_{2}(\tau)-W_{1}(\tau)+W_{2}(\tau) \Delta\right] \text { for } L, \\
{\left[2 W_{1}(\tau)+W_{2}(\tau) \Delta\right] \text { for } T}
\end{array}\right. \\
W_{1}(\tau)= & \tau G_{M}^{2}(\tau), \quad W_{2}(\tau)=\frac{\left[G_{E}^{2}(\tau)+\tau G_{M}^{2}(\tau)\right]}{1+\tau}
\end{aligned}
$$

where $G_{E}$ and $G_{M}$ are the electric and magnetic Sachs form factors, the standard dimensionless variables are defined by

$$
\begin{aligned}
\kappa \equiv q / 2 m_{N}, & \lambda \equiv \omega / 2 m_{N}, \quad \tau=\kappa^{2}-\lambda^{2}, \\
\eta \equiv|\mathbf{p}| / m_{N}, & \varepsilon \equiv E(\mathbf{p}) / m_{N}=\sqrt{1+\eta^{2}}, \\
\eta_{F} & \equiv p_{F} / m_{N}, \quad \varepsilon_{F}=\sqrt{1+\eta_{F}^{2}},
\end{aligned}
$$

and

$\Delta=\frac{\tau}{\kappa^{2}}\left[\frac{1}{3}\left(\varepsilon_{F}^{2}+\varepsilon_{F} \Gamma+\Gamma^{2}\right)+\lambda\left(\varepsilon_{F}+\Gamma\right)+\lambda^{2}\right]-(1+\tau)$,

$\Gamma \equiv \max \left[\left(\varepsilon_{F}-2 \lambda\right), \gamma_{-} \equiv \kappa \sqrt{1+\frac{1}{\tau}}-\lambda\right]$.

In Refs. [18-22] we defined [18,19] and applied the scaling function within the CDFM using the basis of the RFG scaling function. In the model $[15,16]$, the one-body density matrix $\rho\left(\mathbf{r}, \mathbf{r}^{\prime}\right)$ is an infinite superposition of one-body density matrices $\rho_{x}\left(\mathbf{r}, \mathbf{r}^{\prime}\right)$ corresponding to single Slater determinant wave functions of systems of free $A$ nucleons homogeneously distributed in a sphere with radius $x$, density $\rho_{0}(x)=\frac{3 A}{4 \pi x^{3}}$, and Fermi momentum $p_{F}(x)=\left[\frac{3 \pi^{2}}{2} \rho_{0}(x)\right]^{1 / 3}=\alpha / x$ (with $\left.\alpha \approx 1.52 A^{1 / 3}\right)$ :

$$
\rho\left(\mathbf{r}, \mathbf{r}^{\prime}\right)=\int_{0}^{\infty} d x|F(x)|^{2} \rho_{x}\left(\mathbf{r}, \mathbf{r}^{\prime}\right)
$$

The weight function $|F(x)|^{2}$ can be expressed in an equivalent way either by means of the density distribution $[15,16,19]$,

$$
|F(x)|^{2}=-\left.\frac{1}{\rho_{0}(x)} \frac{d \rho(r)}{d r}\right|_{r=x} \text { at } \frac{d \rho(r)}{d r} \leqslant 0,
$$

or by the nucleon momentum distribution [19],

$$
|F(x)|^{2}=-\left.\frac{3 \pi^{2}}{2} \frac{\alpha}{x^{5}} \frac{d n(k)}{d k}\right|_{k=\alpha / x} \text { at } \frac{d n(k)}{d k} \leqslant 0 .
$$

In Eqs. (13) and (14)

$$
\begin{aligned}
\int \rho(\mathbf{r}) d \mathbf{r} & =A, \quad \int n(\mathbf{k}) d \mathbf{k}=A, \\
\int_{0}^{\infty}|F(x)|^{2} d x & =1 .
\end{aligned}
$$

So, in the first version of the CDFM approach the constructed $\left(\mathrm{CDFM}_{\mathrm{I}}\right)$ scaling function has the form $[18,19]$

$$
f\left(\psi^{\prime}\right)=\int_{0}^{\alpha /\left(k_{F}\left|\psi^{\prime}\right|\right)} d x|F(x)|^{2} f_{\mathrm{RFG}}\left(x, \psi^{\prime}\right),
$$


where the RFG scaling function is

$$
\begin{aligned}
f_{\mathrm{RFG}}\left(x, \psi^{\prime}\right)= & \frac{3}{4}\left[1-\left(\frac{k_{F} x\left|\psi^{\prime}\right|}{\alpha}\right)^{2}\right] \\
& \times\left\{1+\left(\frac{x m_{N}}{\alpha}\right)^{2}\left(\frac{k_{F} x\left|\psi^{\prime}\right|}{\alpha}\right)^{2}\right. \\
& \left.\times\left[2+\left(\frac{\alpha}{x m_{N}}\right)^{2}-2 \sqrt{1+\left(\frac{\alpha}{x m_{N}}\right)^{2}}\right]\right\}
\end{aligned}
$$

and the momentum $k_{F}$ is calculated consistently in the CDFM for each nucleus from the expression

$$
k_{F}=\int_{0}^{\infty} d x k_{F}(x)|F(x)|^{2}=\int_{0}^{\infty} d x \frac{\alpha}{x}|F(x)|^{2} .
$$

Thus, $k_{F}$ in the CDFM is not a fitting parameter as it is in the RFG model.

By using Eqs. (13) and (14) in Eqs. (16) and (18) the CDFM scaling function $f\left(\psi^{\prime}\right)$ and $k_{F}$ can be expressed explicitly by the density and momentum distributions [19].

In contrast to the $\mathrm{CDFM}_{\mathrm{I}}$, in this work we construct a more general CDFM approach $\left(\mathrm{CDFM}_{\mathrm{II}}\right)$, starting not from the scaling function, but from the hadronic tensor, the response functions, and related quantities in the model of the RFG with a density $\rho_{0}(x)$ and a Fermi momentum $p_{F}(x)$. Thus, now we replace the quantities $p_{F}, \eta_{F}$, and $\varepsilon_{F}$ in Eqs. (6), (8), and (11) by

$$
\begin{aligned}
& \eta_{F}(x)=\frac{p_{F}(x)}{m_{N}}=\frac{\alpha}{x m_{N}}, \\
& \varepsilon_{F}(x)=\sqrt{1+\eta_{F}^{2}(x)}=\sqrt{1+\left(\frac{\alpha}{x m_{N}}\right)^{2}},
\end{aligned}
$$

and, following the CDFM methods in Refs. [15,16], the hadronic tensor and the response functions in the $\mathrm{CDFM}_{\mathrm{II}}$

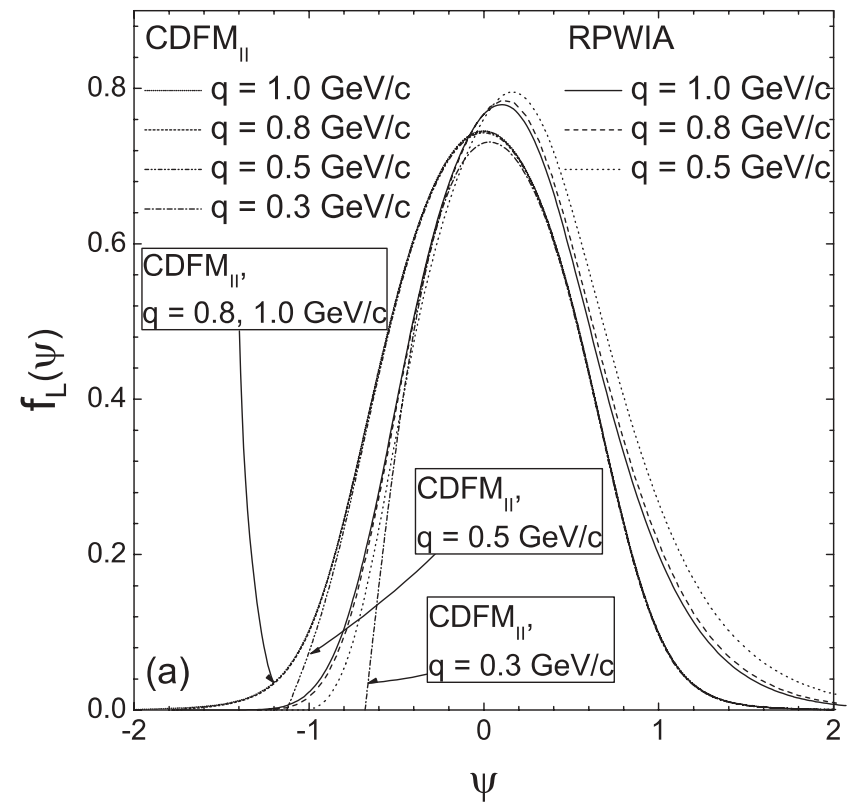

approach are obtained by weighting the RFG model ones by the function $|F(x)|^{2}$ [Eqs. (13) and (14)]:

$$
\begin{aligned}
W_{\mathrm{CDFM}}^{\mu \nu} & =\int_{0}^{\infty}|F(x)|^{2} W_{(\mathrm{RFG})}^{\mu \nu}(x) d x, \\
R_{L}(\psi) & =\int_{0}^{\infty}|F(x)|^{2} R_{L}^{(\mathrm{RFG})}(x, \psi) d x, \\
R_{T}(\psi) & =\int_{0}^{\infty}|F(x)|^{2} R_{T}^{(\mathrm{RFG})}(x, \psi) d x,
\end{aligned}
$$

where $W_{(\mathrm{RFG})}^{\mu \nu}(x)$ and $R_{L, T}^{(\mathrm{RFG})}(x, \psi)$ are given by Eq. (6) and Eq. (8), respectively, but now the Fermi momentum depending on $x$ according to Eq. (19) and the scaling variable $\psi$ is defined by $[1,2]$

$$
\psi \equiv \frac{1}{\sqrt{\xi_{F}}} \frac{\lambda-\tau}{\sqrt{(1+\lambda) \tau+\kappa \sqrt{\tau(1+\tau)}}},
$$

where $\xi_{F}=\sqrt{\left(1+\eta_{F}^{2}\right)}-1$. Note that Eq. (23) is meant to be used only in the Pauli unblocked region $q>2 k_{F}$.

We label $\frac{d^{2} \sigma}{d \Omega d \varepsilon^{\prime}}$ by $C^{\operatorname{CDFM}}(\psi)$ :

$$
\begin{aligned}
C^{\mathrm{CDFM}}(\psi) \equiv & \frac{d^{2} \sigma}{d \Omega d \varepsilon^{\prime}} \\
= & \sigma_{M}\left\{\left(\frac{Q^{2}}{q^{2}}\right)^{2} R_{L}(\psi)+\left[\frac{1}{2}\left|\frac{Q^{2}}{q^{2}}\right|\right.\right. \\
& \left.\left.+\tan ^{2} \frac{\theta}{2}\right] R_{T}(\psi)\right\} .
\end{aligned}
$$

The single-nucleon $e N$ elastic cross section has the form [24]

$$
S=\sigma_{M}\left\{\left(\frac{Q^{2}}{q^{2}}\right)^{2} G_{L}(\tau)+\left[\frac{1}{2}\left|\frac{Q^{2}}{q^{2}}\right|+\tan ^{2} \frac{\theta}{2}\right] G_{T}(\tau)\right\},
$$

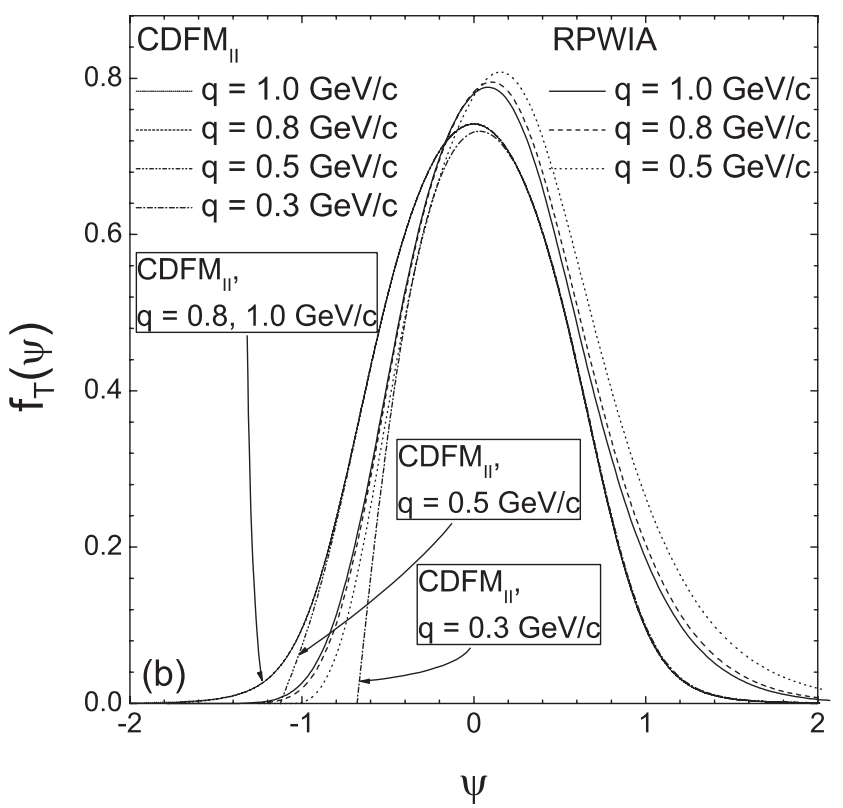

FIG. 1. The longitudinal scaling functions $f_{L}(\psi)$ (a) and the transverse scaling functions $f_{T}(\psi)$ (b) for ${ }^{12} \mathrm{C}$ calculated in the $\mathrm{CDFM}_{\mathrm{II}}$ for $q=0.3,0.5,0.8$, and $1.0 \mathrm{GeV} / c$ and RPWIA (Lorentz gauge) for $q=0.5,0.8$, and $1.0 \mathrm{GeV} / c$. 


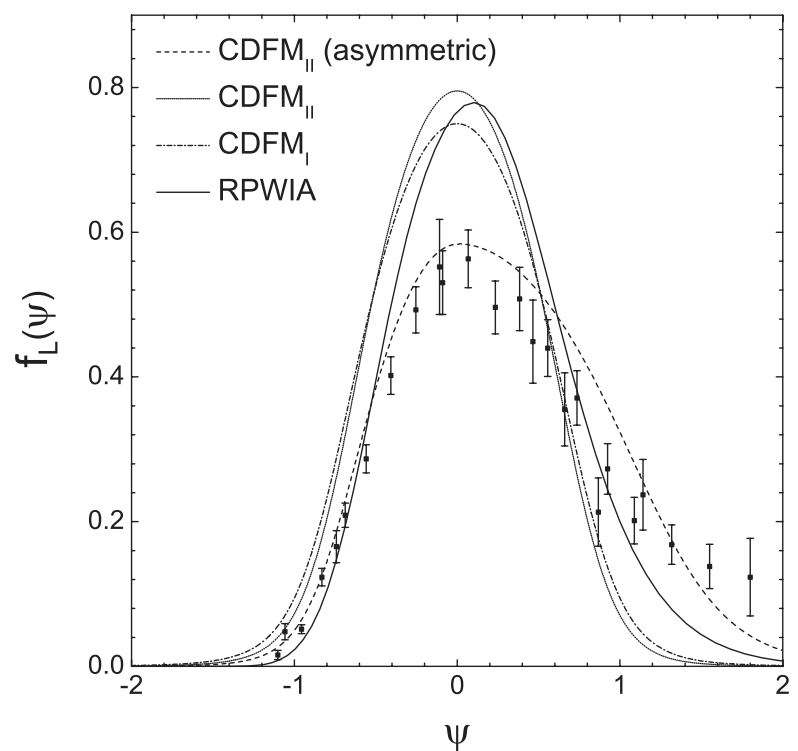

FIG. 2. The longitudinal scaling functions $f_{L}(\psi)$ for ${ }^{12} \mathrm{C}$ calculated for $q=1 \mathrm{GeV} / c$ in the $\mathrm{CDFM}_{\mathrm{I}}, \mathrm{CDFM}_{\mathrm{II}}$, RPWIA, and asymmetric $\mathrm{CDFM}_{\mathrm{II}}$. The experimental data are taken from Ref. [25].

where the single-nucleon functions $G_{L}$ and $G_{T}$ are given by

$$
\begin{aligned}
G_{L}(\tau) & =\frac{\kappa}{2 \tau}\left[Z G_{E, p}^{2}(\tau)+N G_{E, n}^{2}(\tau)\right]+\mathcal{O}\left(\eta_{F}^{2}\right), \\
G_{T}(\tau) & =\frac{\tau}{\kappa}\left[Z G_{M, p}^{2}(\tau)+N G_{M, n}^{2}(\tau)\right]+\mathcal{O}\left(\eta_{F}^{2}\right) .
\end{aligned}
$$

Then the superscaling function can be obtained by

$$
f^{\mathrm{CDFM}_{\mathrm{II}}}(\psi)=p_{F} \times \frac{C^{\mathrm{CDFM}}(\psi)}{S},
$$

and, finally, following Ref. [4] longitudinal $L$ and transverse $T$ scaling functions can be introduced:

$$
f_{L}(\psi)=p_{F} \times \frac{R_{L}(\psi)}{G_{L}},
$$

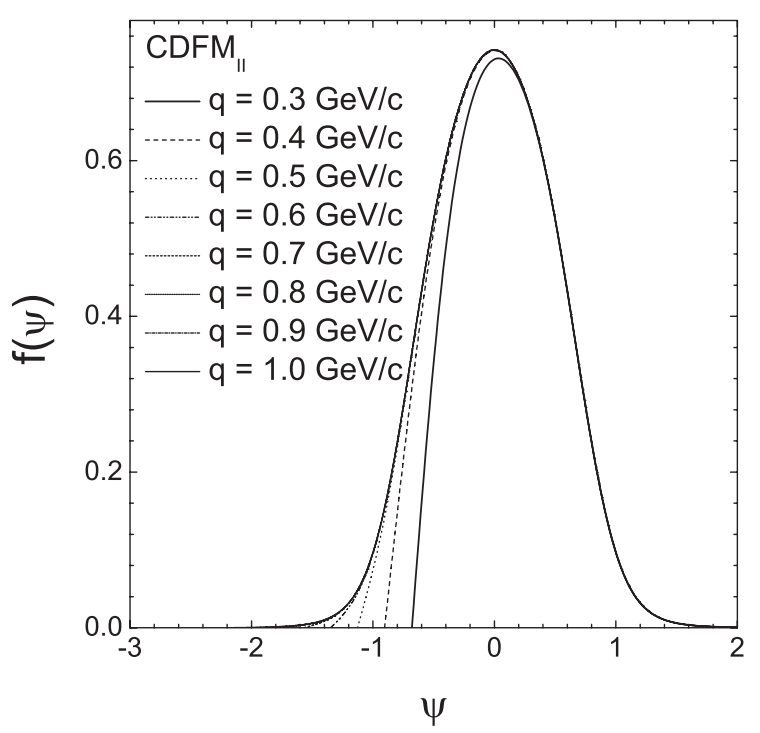

FIG. 3. The quasielastic scaling function $f^{\mathrm{QE}}(\psi)$ for ${ }^{12} \mathrm{C}$ calculated in the $\mathrm{CDFM}_{\mathrm{II}}$ for $q=0.3-1.0 \mathrm{GeV} / c$ with steps of $0.1 \mathrm{GeV} / c$.

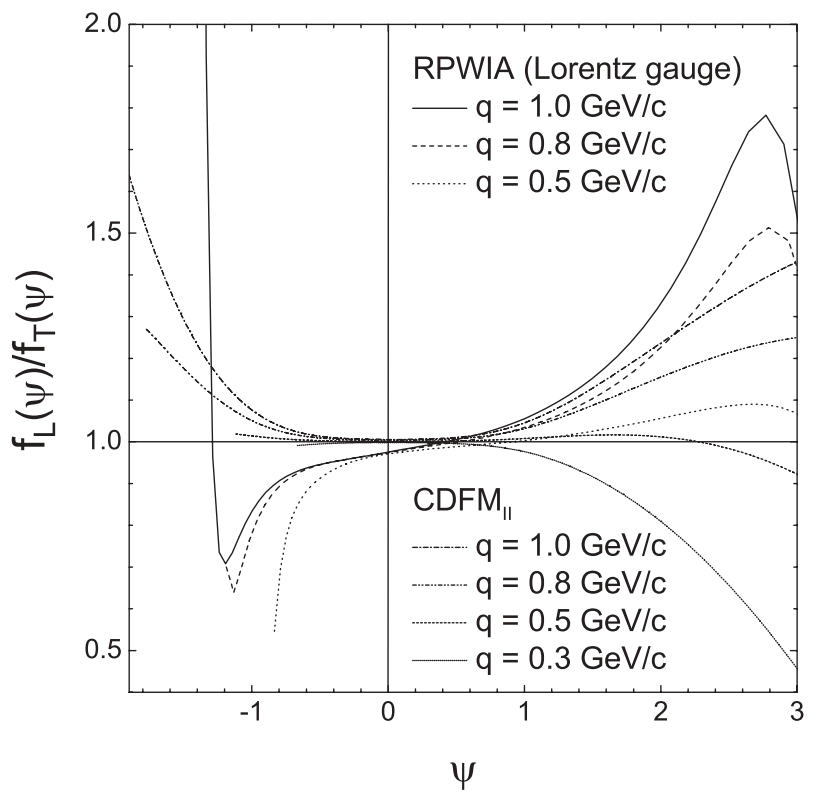

FIG. 4. The ratio $f_{L}(\psi) / f_{T}(\psi)$ for ${ }^{12} \mathrm{C}$ calculated in the $\mathrm{CDFM}_{\mathrm{II}}$ for $q=0.3,0.5,0.8$, and $1.0 \mathrm{GeV} / c$ and RPWIA (Lorentz gauge) for $q=0.5,0.8$, and $1.0 \mathrm{GeV} / c$.

$$
f_{T}(\psi)=p_{F} \times \frac{R_{T}(\psi)}{G_{T}} .
$$

We note that this approach differs from the first version of the CDFM applied to the scaling phenomenon [18-22] where the RFG scaling function $f_{\mathrm{RFG}}\left(\psi^{\prime}, x\right)$ is directly weighted by the function $|F(x)|^{2}$ [Eqs. (16) and (17)].

As mentioned in Sec. I, in this paper we mark the CDFM approach developed in our previous works [14,18-22,56] as $\mathrm{CDFM}_{\mathrm{I}}$ in contrast with the $\mathrm{CDFM}_{\mathrm{II}}$ one presented here in Sec. II. We would like to note that, as can be seen in Sec. III,

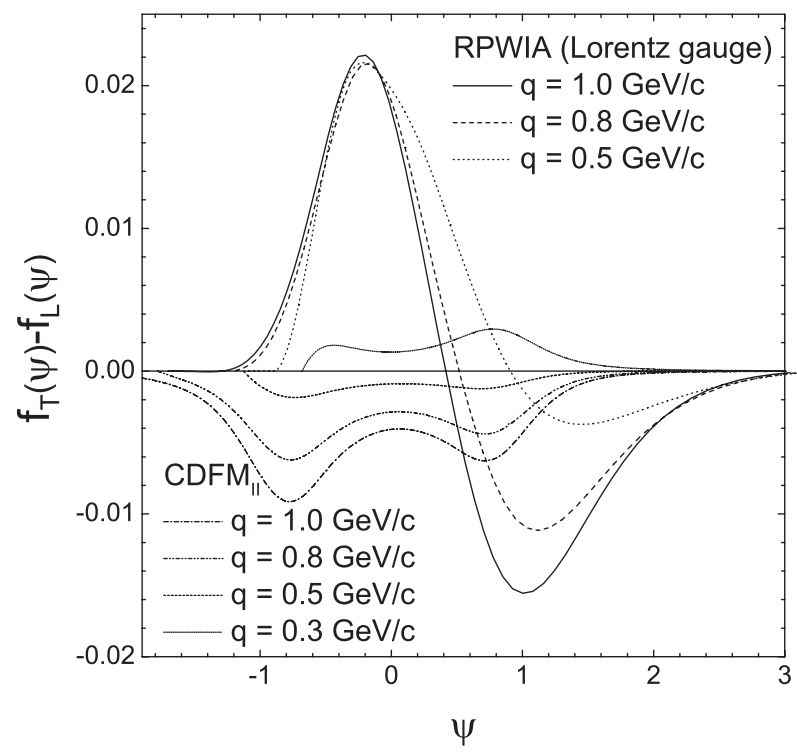

FIG. 5. The differences $f_{T}(\psi)-f_{L}(\psi)$ for ${ }^{12} \mathrm{C}$ calculated in the $\mathrm{CDFM}_{\mathrm{II}}$ for $q=0.3,0.5,0.8$, and $1.0 \mathrm{GeV} / c$ and RPWIA (Lorentz gauge) for $q=0.5,0.8$, and $1.0 \mathrm{GeV} / c$. 

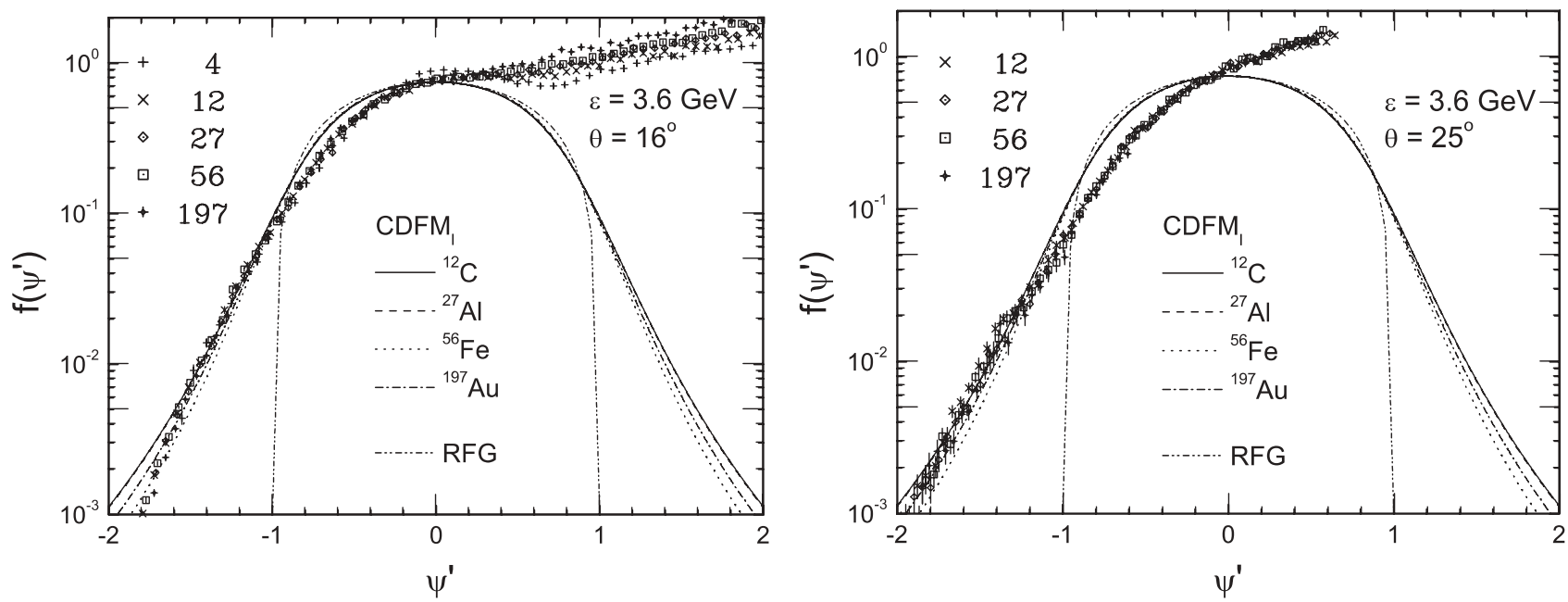

FIG. 6. The quasielastic scaling function $f^{\mathrm{QE}}\left(\psi^{\prime}\right)$ for ${ }^{12} \mathrm{C},{ }^{27} \mathrm{Al},{ }^{56} \mathrm{Fe}$, and ${ }^{197} \mathrm{Au}$ calculated in the $\mathrm{CDFM}_{\mathrm{I}}$ and RFG model. The experimental data are taken from Ref. [3] and the labels indicate the mass number for each set of data.

in the $\mathrm{CDFM}_{\mathrm{II}}, f_{L}^{\mathrm{CDFM}_{\mathrm{II}}}(\psi) \neq f_{T}^{\mathrm{CDFM}_{\mathrm{II}}}(\psi)$, in contrast with $\mathrm{CDFM}_{\mathrm{I}}$, where $f_{L}^{\mathrm{CDFM}_{\mathrm{I}}}(\psi)=f_{T}^{\mathrm{CDFM}_{\mathrm{I}}}(\psi)$. The results and discussions are given in the next section.

\section{RESULTS}

In this section we first present our results of longitudinal [Fig. 1(a)] and transverse [Fig. 1(b)] scaling functions at fixed values of momentum transfer $q=0.3,0.5,0.8$, and $1.0 \mathrm{GeV} / c$ calculated within the $\mathrm{CDFM}_{\mathrm{II}}$ approach compared with results of the RPWIA with Lorentz gauge [37]. In contrast with our previous results, where the $\mathrm{CDFM}_{\mathrm{I}}$ scaling functions are equal, $f_{L}^{\mathrm{CDFM}_{\mathrm{I}}}(\psi)=f_{T}^{\mathrm{CDFM}_{\mathrm{I}}}(\psi)=f^{\mathrm{CDFM}_{\mathrm{I}}}(\psi)$, and do not depend on the momentum transfer $q$, in the $\mathrm{CDFM}_{\mathrm{II}}$ the scaling functions, which are built from the nuclear electromagnetic response functions, depend on the momentum transfer $q$ till a sufficiently high $q$. As can be seen from Fig. 1, scaling of the first kind is clearly violated for low $q$ values $(q<$ $0.5 \mathrm{GeV} / c$ ) in the negative $\psi$ region, whereas for $q$ of the order of $0.5 \mathrm{GeV} / c$, scaling violation slowly disappears as $q$ increases and the $\mathrm{CDFM}_{\mathrm{II}}$ and RPWIA scaling functions reach their asymptotic values. In Fig. 2 we give a comparison of the longitudinal scaling functions from $\mathrm{CDFM}_{\mathrm{I}}, \mathrm{CDFM}_{\mathrm{II}}$, and RPWIA with the experimental data. We note that all three approaches overestimate the data, especially at the QE peak and in the high positive $\psi$ region. A better comparison with data can be achieved by introducing, as done in our previous work [21], a phenomenological asymmetric tail for $\psi>0$ in such a way to preserve the correct normalization of the superscaling function: The corresponding result, labeled "CDFM ${ }_{\text {II }}$ (asymmetric)," is also shown in the figure. Similar behavior for the total QE scaling function can be seen in Fig. 3, where we present our results of calculations of $f^{\mathrm{QE}}(\psi)$ [Eq. (28)] for ${ }^{12} \mathrm{C}$ within the $\mathrm{CDFM}_{\mathrm{II}}$ for $q=0.3-1.0 \mathrm{GeV} / c$ with steps of $0.1 \mathrm{GeV} / c$. Note that the asymmetry in the
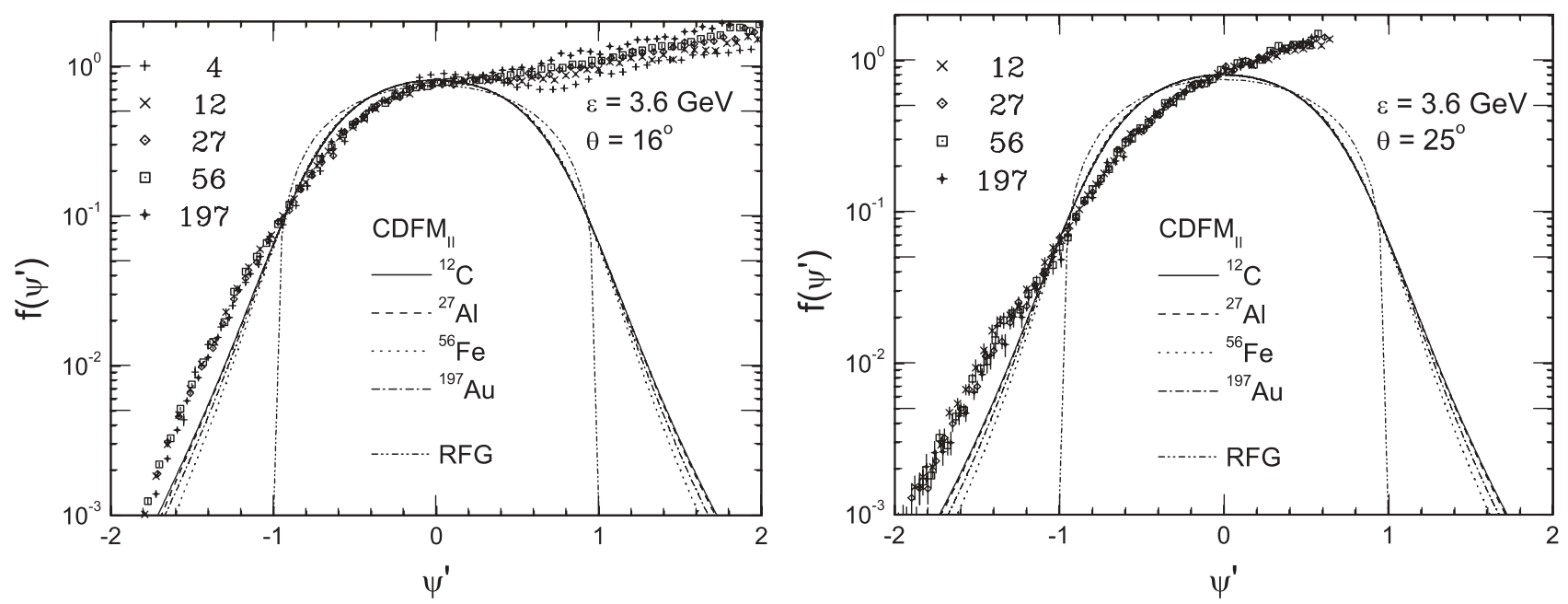

FIG. 7. The quasielastic scaling function $f^{\mathrm{QE}}\left(\psi^{\prime}\right)$ for ${ }^{12} \mathrm{C},{ }^{27} \mathrm{Al},{ }^{56} \mathrm{Fe}$, and ${ }^{197} \mathrm{Au}$ calculated in the CDFM $\mathrm{II}$ and RFG model. The experimental data are taken from Ref. [3] and the labels indicate the mass number for each set of data. 


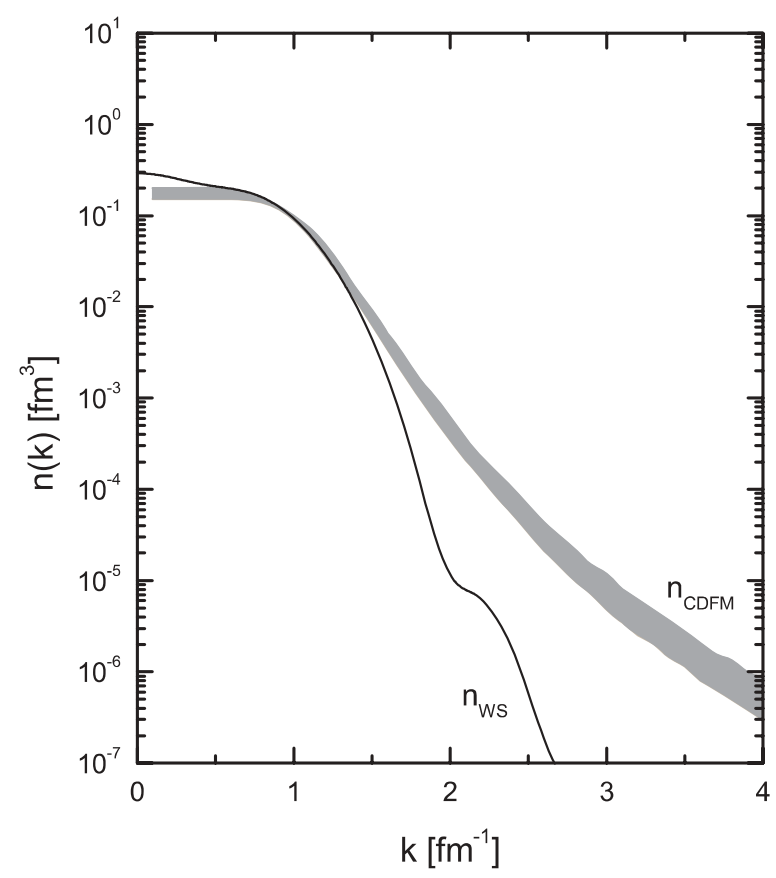

FIG. 8. The nucleon momentum distribution $n(k)$ (also see Fig. 2 of Ref. [19] and Fig. 3 of Ref. [21]). The gray area shows the combined results of CDFM for ${ }^{4} \mathrm{He},{ }^{12} \mathrm{C},{ }^{27} \mathrm{Al},{ }^{56} \mathrm{Fe}$, and ${ }^{197} \mathrm{Au}$. The solid line is the mean-field result using Woods-Saxon single-particle wave functions (for ${ }^{56} \mathrm{Fe}$ ). The normalization is $\int n(k) d^{3} k=1$.

scaling function, clearly observed for low $q$ values, tends to disappear as $q$ goes up. This asymmetry in the negative $\psi$ region at low $q$ is linked to effects introduced by Pauli blocking, which destroy the scaling behavior. We note that the results of $\mathrm{CDFM}_{\mathrm{II}}$ at $q=0.8 \mathrm{GeV} / c$ and $q=1.0 \mathrm{GeV} / c$ are similar among themselves in both the negative and positive $\psi$ regions.

In Figs. 4 and 5 we present results for the ratio $f_{L}(\psi) / f_{T}(\psi)$ and the differences $f_{T}(\psi)-f_{L}(\psi)$ for ${ }^{12} \mathrm{C}$ calculated in the $\mathrm{CDFM}_{\mathrm{II}}$ and RPWIA (Lorentz gauge) at fixed values of momentum transfer $q=0.3,0.5,0.8$, and $1.0 \mathrm{GeV} / c$. In the $\mathrm{CDFM}_{\text {II }}$ calculations we observe violation of the scaling of the zeroth kind $\left[f_{L}(\psi) \neq f_{T}(\psi)\right]$, at variance with the $\mathrm{CDFM}_{\mathrm{I}}$ one. The behavior of the ratio $f_{L}(\psi) / f_{T}(\psi)$ in our model is similar to that in the RPWIA for positive $\psi$ values where the response is positive except for very low $q(q=$ $0.3 \mathrm{GeV} / c)$. In contrast, in the negative $\psi$ region, the ratio $f_{L}(\psi) / f_{T}(\psi)$ becomes negative for RPWIA and positive for $\mathrm{CDFM}_{\mathrm{II}}$, the variation in the former case being much larger. These results are consistent with the ones shown in Fig. 5. Here, the difference $f_{T}(\psi)-f_{L}(\psi)$ is negative (positive) for all $q$ values $(q=0.3 \mathrm{GeV} / c)$ in the whole $\psi$ region in the case of the $\mathrm{CDFM}_{\mathrm{II}}$. This is in contrast with RPWIA results where $f_{T}(\psi)-f_{L}(\psi)$ starts being positive (left $\psi$ region), changing to negative for higher $\psi$. The specific value of $\psi$ where $f_{T}(\psi)-f_{L}(\psi)$ changes sign depends on the $q$ value considered, being larger as $q$ increases. As a general outcome, we conclude that $\mathrm{CDFM}_{\mathrm{II}}$ scaling functions are not so different from each other as they are in the RPWIA case.
The next step in our studies is to examine the scaling of the second kind in the $\mathrm{CDFM}_{\mathrm{II}}$. This requires calculations of the scaling functions for different nuclei. In Figs. 6 and 7 we give the results for the $\mathrm{QE}$ scaling functions for ${ }^{12} \mathrm{C}$, ${ }^{27} \mathrm{Al},{ }^{56} \mathrm{Fe}$, and ${ }^{197} \mathrm{Au}$ calculated in the $\mathrm{CDFM}_{\mathrm{I}}$ and $\mathrm{CDFM}_{\mathrm{II}}$, respectively. The result of the RFG model is also presented. One can see the essential difference between the results of the RFG model and those of the $\mathrm{CDFM}_{\mathrm{I}}$ and $\mathrm{CDFM}_{\mathrm{II}}$ in the region $\psi^{\prime}<-1$. For readers who may not be familiar with the scaling variable $\psi^{\prime}$ we recall that the variable $\psi$, first introduced by Alberico et al. [1] as the natural scaling variable within the RFG model, is defined [see Eq. (23)] so that it varies from $\psi \sim\left(-\kappa / \sqrt{\xi_{F}}\right)$ to $\psi=0$ on the left-hand side of the quasielastic peak (i.e., when the transfer energy $\omega$ varies from 0 to $Q^{2} / 2 m_{N}$ ), whereas $\psi>0$ when we are in the right-hand side and other production channels may start to open. The variable $\psi^{\prime}$ was introduced in Refs. [11] and [3,4] as the corresponding phenomenological variable to analyze data and to show scaling of the second kind. It involves a redefinition of $\lambda$ that corrects for the displacement in $\omega$ of the QE peak position, which depends on the nuclear target. It can be seen also from our results that the scaling of the second kind is good in both CDFM approaches; however, the $\mathrm{CDFM}_{\mathrm{I}}$ scaling functions are in better agreement with the experimental data. This is because the maximum of $f^{\mathrm{CDFM}_{\mathrm{II}}}(\psi)$ is 0.80 (coming from the expressions for the RFG hadronic tensor) but not 0.75 as it is in the RFG model and, correspondingly, in the CDFM ${ }_{I}$. In this case

$$
f_{\max }^{\mathrm{CDFM}_{\mathrm{II}}}(\psi) \approx 0.8>f_{\max }^{\mathrm{CDFM}_{\mathrm{I}}}(\psi)=0.75
$$

and the normalization

$$
\int_{-\infty}^{\infty} f^{\mathrm{CDFM}_{\mathrm{II}}}(\psi) d \psi=\int_{-\infty}^{\infty} f^{\mathrm{CDFM}_{\mathrm{I}}}(\psi) d \psi=1
$$

leads to narrower behavior of $f(\psi)$ in the $\mathrm{CDFM}_{\mathrm{II}}$.

The behavior of the $\mathrm{CDFM}_{\mathrm{I}}$ and $\mathrm{CDFM}_{\mathrm{II}}$ scaling functions can be explained by the long-range collective correlations included in the CDFM, which is based on the generator coordinate method [17] applied to consider the monopole breathing motions $[15,16]$. These correlations are important and they are reflected in the tail of the CDFM scaling functions at negative $\psi^{\prime}$. In contrast, the results of mean-field approaches (relativistic or not) are generally closer to those of the RFG model. Although the differences of the results of $\mathrm{CDFM}_{\mathrm{I}}$ and $\mathrm{CDFM}_{\mathrm{II}}$ are not large, they reflect the different stage at which the RFG approach is replaced by the CDFM (using the weight function): In the $\mathrm{CDFM}_{\text {II }}$ long-range correlations are included at the level of the hadron tensor, whereas in the $\mathrm{CDFM}_{\mathrm{I}}$ this is done directly in the scaling function after having factorized and divided by the single-nucleon factors.

To illustrate the effects of the $N N$ correlations included in the CDFM on the tail of the CDFM scaling function we recall the relationship (mentioned in Sec. I) between the scaling function $f\left(\psi^{\prime}\right)$ and the nucleon momentum distribution $n(k)$. It was found within the CDFM [14,18,19,21] that the slope of the QE scaling function $f\left(\psi^{\prime}\right)$ at negative $\psi^{\prime}$ crucially depends on the high-momentum tail of $n(k)$ at larger values of $k(k>$ $\left.1.5\left[\mathrm{fm}^{-1}\right]\right)$. This can be seen in Fig. 8 from the difference 

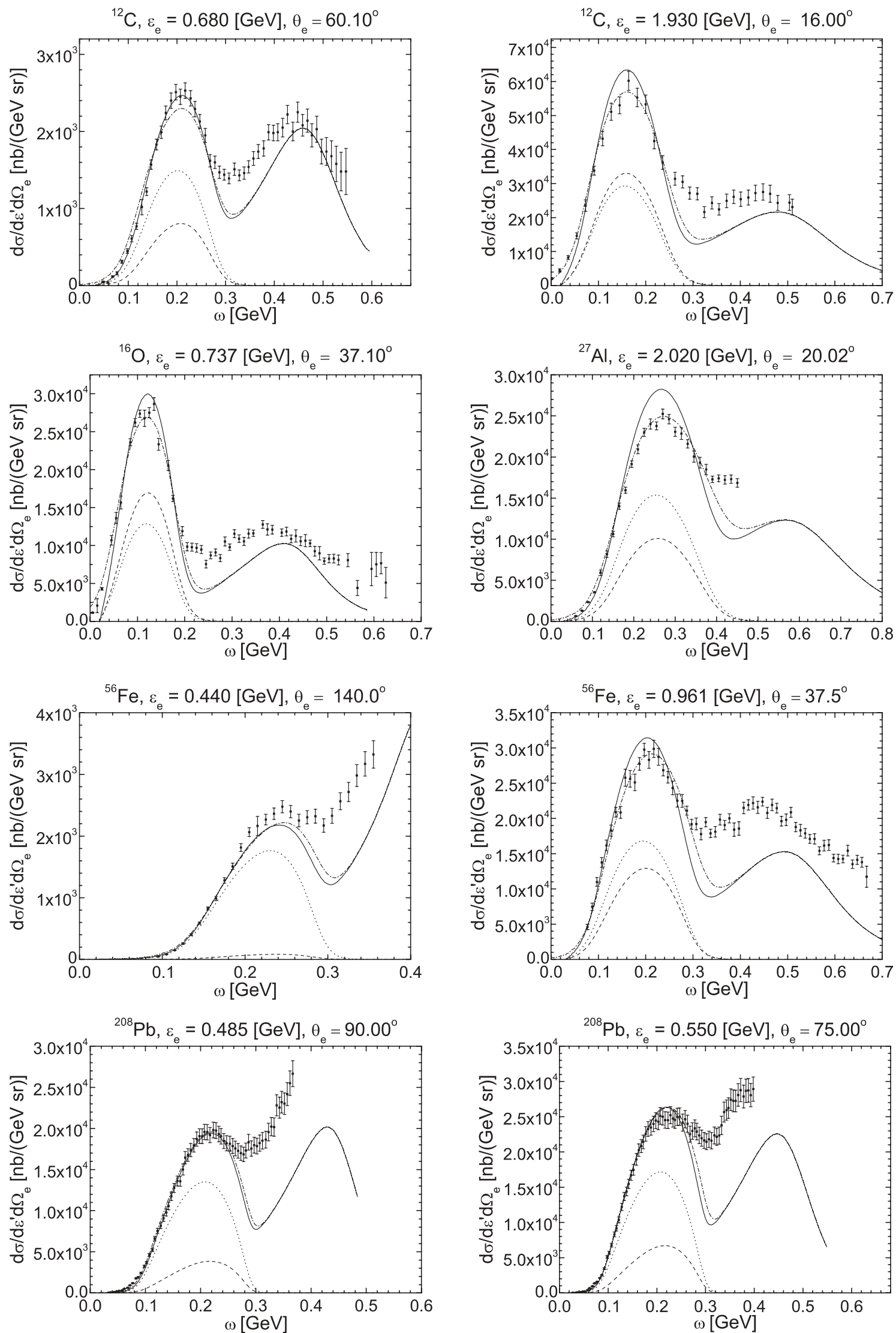

FIG. 9. Inclusive electron cross sections as function of energy loss. Shown are results for the $\mathrm{CDFM}_{\mathrm{I}}$ (dash-dotted line), the CDFM $\mathrm{II}_{\text {( }}$ (solid line), the $L$ contribution in the $\mathrm{CDFM}_{\mathrm{II}}$ (dashed line), and the $T$ contribution in the $\mathrm{CDFM}_{\mathrm{II}}$ (dotted line). The experimental data are taken from Ref. [26]. 


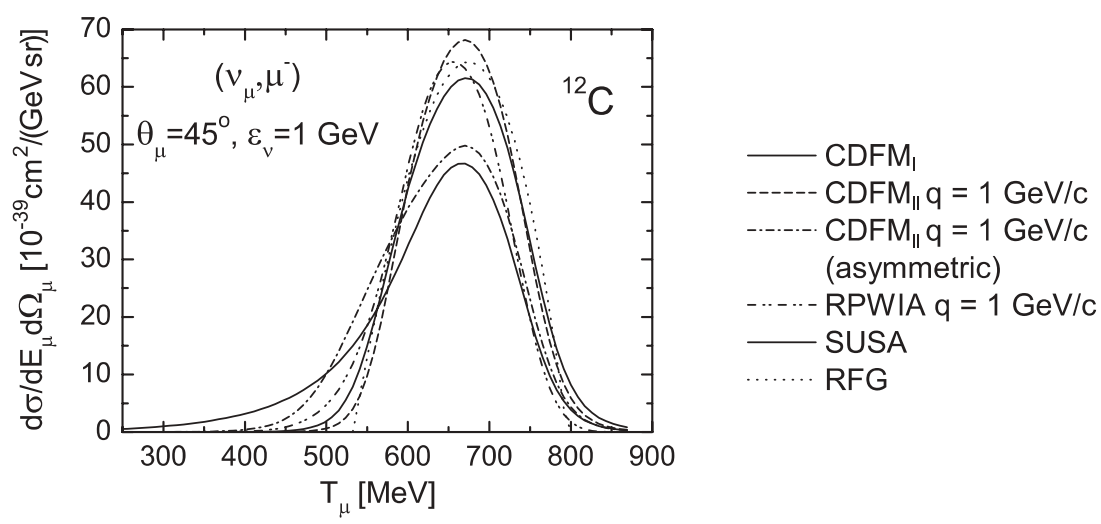

FIG. 10. The cross section of the chargechanging neutrino $\left(v_{\mu}, \mu^{-}\right)$reaction on ${ }^{12} \mathrm{C}$ at $\theta_{\mu}=45^{\circ}$ and $\varepsilon_{v}=1 \mathrm{GeV}$. between the CDFM combined results $\left(n_{\mathrm{CDFM}}\right)$ for ${ }^{4} \mathrm{He},{ }^{12} \mathrm{C}$, ${ }^{27} \mathrm{Al},{ }^{56} \mathrm{Fe}$, and ${ }^{197} \mathrm{Au}$ (gray area) and the mean-field result ( $n_{\text {WS }}$ ) obtained by means of Woods-Saxon single-particle wave functions (for ${ }^{56} \mathrm{Fe}$ ). It was shown in Refs. $[18,19]$ that when the scaling function is calculated using realistic high-momentum components of $n(k)$ at $k>1.5\left[\mathrm{fm}^{-1}\right]$ (i.e., obtained in a nuclear model accounting for $N N$ correlations beyond the mean-field approximation), a reasonable explanation of the superscaling behavior of the scaling function for $\psi^{\prime}<-1$ is achieved. We note that the difference between the CDFM scaling function and that from the RFG model for $\left|\psi^{\prime}\right|>1$, which can be seen in Figs. 6 and 7, is due to the large difference between $n(k)$ in the CDFM and that in the RFG model, where the (dimensionless) momentum distribution is a step function. The study performed in Ref. [19] of the sensitivity of the CDFM scaling function to the particular behavior of $n(k)$ in different regions of $k$ showed that the available empirical data on $f\left(\psi^{\prime}\right)$ are informative for $n(k)$ for momentum $k \leqslant 2.0-2.5\left[\mathrm{fm}^{-1}\right]$.

A test of the CDFM superscaling functions is performed (Fig. 9) by calculations of the cross sections of electron scattering in QE and $\Delta$ regions for nuclei with $12 \leqslant A \leqslant 208$ at different energies and angles using the $\mathrm{CDFM}_{\mathrm{I}}$ and $\mathrm{CDFM}_{\mathrm{II}}$ scaling functions. For the scaling function in the $\Delta$ region we use the results of Ref. [19]. The results are compared with available experimental data.

As can be seen from Fig. 9 the results calculated with both $\mathrm{CDFM}_{\mathrm{I}}$ and $\mathrm{CDFM}_{\mathrm{II}}$ scaling functions do not differ too much, agreeing well with experimental data in the QE region. In some particular cases, $\mathrm{CDFM}_{\mathrm{II}}$ overestimates data whereas $\mathrm{CDFM}_{\mathrm{I}}$ agrees better, being the reverse in other situations. Finally, some kinematical regimes lead to very similar results for both models, being in excellent accord with data. Away from the QE and $\Delta$ peaks the behavior of the cross sections is due to higher resonances and, as can be expected, in some cases our results are not in good agreement with the experimental data. We also display the separate longitudinal and transverse contributions to the QE peak.

Finally, in Fig. 10 we present the CDFM results for the cross section of the charge-changing neutrino $\left(v_{\mu}, \mu^{-}\right)$reaction on ${ }^{12} \mathrm{C}$ at $\theta_{\mu}=45^{\circ}$ and $\varepsilon_{v}=1 \mathrm{GeV}$. The calculations are performed following the formalism from Refs. [21,24] using not only the $\mathrm{CDFM}_{\mathrm{I}}$ but also the $\mathrm{CDFM}_{\mathrm{II}} \mathrm{QE}$ scaling function. Our results are compared with those from the RFG model and SuSA and RPWIA approaches.
We note that the CDFM results are qualitatively similar to that of Fig. 2; namely, the result for the $\mathrm{CDFM}_{\mathrm{II}}$ with asymmetry is closer to that calculated using the phenomenological (SuSA) scaling function that is extracted from the experimental data on inclusive electron scattering. However, $\mathrm{CDFM}_{\mathrm{I}}$ and $\mathrm{CDFM}_{\mathrm{II}}$ models lead to very close results, with the maximum of the scaling function being slightly higher in the latter. The scaling functions for both approaches follow closely the behavior exhibited by the RPWIA one.

\section{CONCLUSIONS}

The results of the present work can be summarized as follows:

(i) A new, more general, approach within the coherent density fluctuation model is proposed $\left(\mathrm{CDFM}_{\mathrm{II}}\right)$. We apply it to calculate the total $f\left(\psi^{\prime}\right)$, the longitudinal $f_{L}\left(\psi^{\prime}\right)$, and the transverse $f_{T}\left(\psi^{\prime}\right)$ scaling functions by taking as starting point the hadronic tensor and the longitudinal and transverse response functions in the RFG model.

(ii) The approach leads to a slight violation of the zero-kind scaling $\left[f_{L}\left(\psi^{\prime}\right) \neq f_{T}\left(\psi^{\prime}\right)\right]$ in contrast with the situation in the RFG model and CDFM .

(iii) It is found that the ratio $f_{L}\left(\psi^{\prime}\right) / f_{T}\left(\psi^{\prime}\right)$ in the $\mathrm{CDFM}_{\mathrm{II}}$ has similarities with that from the RPWIA approach (with Lorentz gauge) for positive $\psi^{\prime}$.

(iv) It is shown that the $\mathrm{CDFM}_{\mathrm{II}}$ scaling functions calculated for different values of the transferred momentum $q$ show a saturation of its asymptotic behavior. Scaling of the first kind appears at $q$ larger than $\approx 0.5 \mathrm{GeV} / c$.

(v) The CDFM scaling functions are applied to calculate cross sections of inclusive electron scattering (and their longitudinal and transverse components) in the quasielastic and $\Delta$ regions for nuclei with $12 \leqslant A \leqslant 208$ at different energies and angles. The results are in good agreement with available experimental data, especially in the QE region.

(vi) The $\mathrm{CDFM}_{\mathrm{II}}$ approach is applied to calculate chargechanging neutrino (antineutrino) scattering on ${ }^{12} \mathrm{C}$ at $1 \mathrm{GeV}$ incident energy. The results are compared with those from the RFG model, as well as from the SuSA and RPWIA approaches. 


\section{ACKNOWLEDGMENTS}

This work was partly supported by the Bulgarian National Science Fund under Contracts Nos. DO 02-285 and $\Phi$ 1501, by Ministerio de Educación y Ciencia (Spain) under Contract Nos. FPA2006-13807-C02-01, FIS2005-01105, FIS2005-00640, FIS2008-04189, and PCI2006-A7-0548, and by the Spanish Consolider-Ingenio 2010 programme CPAN
(CSD2007-00042). This work is also partially supported by the EU program ILIAS N6 ENTApP WP1. M.V.I. acknowledges support from the European Operational programm HRD through Contract No. BGO051PO001/07/3.3-02/53 with the Bulgarian Ministry of Education. M.B.B. and J.A.C. acknowledge support from the INFN-MEC agreement, project "Study of relativistic dynamics in neutrino and electron scattering."
[1] W. M. Alberico, A. Molinari, T. W. Donnelly, E. L. Kronenberg, and J. W. Van Orden, Phys. Rev. C 38, 1801 (1988).

[2] M. B. Barbaro, R. Cenni, A. De Pace, T. W. Donnelly, and A. Molinari, Nucl. Phys. A643, 137 (1998).

[3] T. W. Donnelly and I. Sick, Phys. Rev. Lett. 82, 3212 (1999).

[4] T. W. Donnelly and I. Sick, Phys. Rev. C 60, 065502 (1999).

[5] G. B. West, Phys. Rep. 18, 263 (1975).

[6] I. Sick, D. B. Day, and J. S. McCarthy, Phys. Rev. Lett. 45, 871 (1980).

[7] C. Ciofi degli Atti, E. Pace, and G. Salmè, Phys. Rev. C 36, 1208 (1987).

[8] D. B. Day, J. S. McCarthy, T. W. Donnelly, and I. Sick, Annu. Rev. Nucl. Part. Sci. 40, 357 (1990).

[9] C. Ciofi degli Atti, E. Pace, and G. Salmè, Phys. Rev. C 43, 1155 (1991).

[10] C. Ciofi degli Atti and S. Simula, Phys. Rev. C 53, 1689 (1996).

[11] R. Cenni, T. W. Donnelly, and A. Molinari, Phys. Rev. C 56, 276 (1997).

[12] C. Ciofi degli Atti and G. B. West, Phys. Lett. B458, 447 (1999).

[13] D. Faralli, C. Ciofi degli Atti, and G. B. West, in Proceedings of 2nd International Conference on Perspectives in Hadronic Physics, ICTP, Trieste, Italy, 1999, edited by S. Boffi, C. Ciofi degli Atti, and M. M. Giannini (World Scientific, Singapore, 2000), p. 75.

[14] A. N. Antonov, M. V. Ivanov, M. K. Gaidarov, and E. Moya de Guerra, Phys. Rev. C 75, 034319 (2007).

[15] A. N. Antonov, V. A. Nikolaev, and I. Zh. Petkov, Bulg. J. Phys. 6, 151 (1979); Z. Phys. A 297, 257 (1980); 304, 239 (1982); Nuovo Cimento A 86, 23 (1985); 102, 1701 (1989); A. N. Antonov, D. N. Kadrev, and P. E. Hodgson, Phys. Rev. C 50, 164 (1994).

[16] A. N. Antonov, P. E. Hodgson, and I. Zh. Petkov, Nucleon Momentum and Density Distributions in Nuclei (Clarendon Press, Oxford, 1988); Nucleon Correlations in Nuclei (SpringerVerlag, Berlin-Heidelberg-New York, 1993).

[17] J. J. Griffin and J. A. Wheeler, Phys. Rev. 108, 311 (1957).

[18] A. N. Antonov, M. K. Gaidarov, D. N. Kadrev, M. V. Ivanov, E. Moya de Guerra, and J. M. Udias, Phys. Rev. C 69, 044321 (2004).

[19] A. N. Antonov, M. K. Gaidarov, M. V. Ivanov, D. N. Kadrev, E. Moya de Guerra, P. Sarriguren, and J. M. Udias, Phys. Rev. C 71, 014317 (2005).

[20] A. N. Antonov, M. V. Ivanov, M. K. Gaidarov, E. Moya de Guerra, P. Sarriguren, and J. M. Udias, Phys. Rev. C 73, 047302 (2006).

[21] A. N. Antonov, M. V. Ivanov, M. K. Gaidarov, E. Moya de Guerra, J. A. Caballero, M. B. Barbaro, J. M. Udias, and P. Sarriguren, Phys. Rev. C 74, 054603 (2006).

[22] M. V. Ivanov, M. B. Barbaro, J. A. Caballero, A. N. Antonov, E. Moya de Guerra, and M. K. Gaidarov, Phys. Rev. C 77, 034612 (2008).
[23] M. B. Barbaro, J. A. Caballero, T. W. Donnelly, and C. Maieron, Phys. Rev. C 69, 035502 (2004).

[24] J. E. Amaro, M. B. Barbaro, J. A. Caballero, T. W. Donnelly, A. Molinari, and I. Sick, Phys. Rev. C 71, 015501 (2005); M. B. Barbaro, Nucl. Phys. B, Proc. Suppl. 159, 186 (2006); M. B. Barbaro, J. E. Amaro, J. A. Caballero, and T. W. Donnelly, in Nuclear Theory: Proceedings of 25th International Workshop on Nuclear Theory, Rila Mountains, Bulgaria, June 26-July 1, 2006, edited by S. Dimitrova (DioMira, Sofia, 2006), p. 73.

[25] C. Maieron, T. W. Donnelly, and I. Sick, Phys. Rev. C 65, 025502 (2002).

[26] O. Benhar, D. Day, and I. Sick, Rev. Mod. Phys. 80, 189 (2008)

[27] L. Alvarez-Ruso, M. B. Barbaro, T. W. Donnelly, and A. Molinari, Nucl. Phys. A724, 157 (2003).

[28] J. E. Amaro, M. B. Barbaro, J. A. Caballero, T. W. Donnelly, and A. Molinari, Nucl. Phys. A697, 388 (2002); A723, 181 (2003); Phys. Rep. 368, 317 (2002).

[29] A. De Pace, M. Nardi, W. M. Alberico, T. W. Donnelly, and A. Molinari, Nucl. Phys. A726, 303 (2003); A741, 249 (2004).

[30] J. E. Amaro, M. B. Barbaro, J. A. Caballero, T. W. Donnelly, and A. Molinari, Nucl. Phys. A643, 349 (1998).

[31] J. E. Amaro, M. B. Barbaro, J. A. Caballero, T. W. Donnelly, and A. Molinari, Nucl. Phys. A723, 181 (2003).

[32] J. E. Amaro, M. B. Barbaro, J. A. Caballero, and T. W. Donnelly, Phys. Rev. C 73, 035503 (2006).

[33] M. C. Martinez, J. A. Caballero, T. W. Donnelly, and J. M. Udias, Phys. Rev. Lett. 100, 052502 (2008).

[34] M. C. Martinez, J. A. Caballero, T. W. Donnelly, and J. M. Udias, Phys. Rev. C 77, 064604 (2008).

[35] M. B. Barbaro, R. Cenni, T. W. Donnelly, and A. Molinari, Phys. Rev. C 78, 024602 (2008).

[36] J. A. Caballero, J. E. Amaro, M. B. Barbaro, T. W. Donnelly, C. Maieron, and J. M. Udias, Phys. Rev. Lett. 95, 252502 (2005).

[37] J. A. Caballero, Phys. Rev. C 74, 015502 (2006).

[38] J. E. Amaro, M. B. Barbaro, J. A. Caballero, T. W. Donnelly, and J. M. Udias, Phys. Rev. C 75, 034613 (2007); J. E. Amaro, M. B. Barbaro, J. A. Caballero, and T. W. Donnelly, Phys. Rev. Lett. 98, 242501 (2007).

[39] Y. Fukuda et al. (Super-Kamiokande Collaboration), Phys. Rev. Lett. 81, 1562 (1998); M. H. Ahn et al. (K2K Collaboration), ibid. 90, 041801 (2003); Q.-R. Ahmad et al. (SNO Collaboration), ibid. 87, 071301 (2001); 89, 011301 (2002); K. Eguchi et al. (KamLAND Collaboration), ibid. 90, 021802 (2003); C. Athanassopoulos et al. (LSND Collaboration), ibid. 77, 3082 (1996); 81, 1774 (1998).

[40] J. E. Amaro, M. B. Barbaro, J. A. Caballero, T. W. Donnelly, and C. Maieron, Phys. Rev. C 71, 065501 (2005). 
[41] J. A. Caballero, J. E. Amaro, M. B. Barbaro, T. W. Donnelly, and J. M. Udias, Phys. Lett. B653, 366 (2007).

[42] M. C. Martinez, P. Lava, N. Jachowicz, J. Ryckebusch, K. Vantournhout, and J. M. Udias, Phys. Rev. C 73, 024607 (2006).

[43] J. Nieves, M. Valverde, and M. J. Vicente-Vacas, Nucl. Phys. B, Proc. Suppl. 155, 263 (2006); nucl-th/0510010; Phys. Rev. C 73, 025504 (2006).

[44] M. B. Barbaro, J. E. Amaro, J. A. Caballero, T. W. Donnelly, and A. Molinari, Nucl. Phys. B, Proc. Suppl. 155, 257 (2006); nucl-th/0509022.

[45] C. Maieron, M. C. Martinez, J. A. Caballero, and J. M. Udias, Phys. Rev. C 68, 048501 (2003).

[46] A. Meucci, C. Giusti, and F. D. Pacati, Nucl. Phys. A739, 277 (2004); A773, 250 (2006).

[47] O. Benhar, Nucl. Phys. B, Proc. Suppl. 139, 15 (2005); nuclth/0408045; O. Benhar and N. Farina, Nucl. Phys. B, Proc. Suppl. 139, 230 (2005); nucl-th/0407106; O. Benhar, N. Farina, H. Nakamura, M. Sakuda, and R. Seki, Nucl. Phys. B, Proc. Suppl. 155, 254 (2006); hep-ph/0510259; Phys. Rev. D 72, 053005 (2005).
[48] G. Co', Nucl. Phys. B, Proc. Suppl. 159, 192 (2006); nuclth/0601034; A. Botrugno and G. Co', Nucl. Phys. A761, 200 (2005); M. Martini, G. Co', M. Anguiano, and A. M. Lallena, Phys. Rev. C 75, 034604 (2007).

[49] T. Leitner, L. Alvarez-Ruso, and U. Mosel, Phys. Rev. C 73, 065502 (2006).

[50] B. Szczerbinska, T. Sato, K. Kubodera, and T.-S. H. Lee, Phys. Lett. B649, 132 (2007).

[51] O. Buss, T. Leitner, U. Mosel, and L. Alvarez-Ruso, Phys. Rev. C 76, 035502 (2007).

[52] A. Meucci, C. Giusti, and F. D. Pacati, Nucl. Phys. A744, 307 (2004)

[53] W. M. Alberico and C. Maieron, arXiv:hep-ph/0210017.

[54] T. Leitner, L. Alvarez-Ruso, and U. Mosel, Phys. Rev. C 74, 065502 (2006).

[55] K. S. Kim, B. G. Yu, M. K. Cheoun, T. K. Choi, and M. T. Chung, J. Phys. G: Nucl. Part. Phys. 34, 2643 (2007); arXiv:0707.1370 [nucl-th].

[56] A. N. Antonov, M. V. Ivanov, M. B. Barbaro, J. A. Caballero, E. Moya de Guerra, and M. K. Gaidarov, Phys. Rev. C 75, 064617 (2007). 